Sección Básica / Basic

Artículos de investigación / Research paper

\title{
El barrenador del fruto y tallo del cacao (Carmenta theobromae, Lepidoptera: Sesiidae) en el valle de Zarumilla, Tumbes, Perú
}

\author{
The fruit borer and the cacao stem (Carmenta theobromae, Lepidoptera: Sesiidae) \\ in the Zarumilla valley, Tumbes, Peru
}

\author{
JOSÉ LUIS MORÁN-ROSILLO ${ }^{1}$; PEDRO SAÚL CASTILLO-CARRILLO²
}

\begin{abstract}
${ }^{1}$ Ing. Agr. Gobierno Regional de Tumbes, Tumbes, Perú, josemoranrosillo@gmail.com, https://orcid.org/0000-0003-4740-593X. ${ }^{2}$ Dr. Jefe del Museo de Entomología y Profesor Principal, Facultad de Ciencias Agrarias, Universidad Nacional de Tumbes, Tumbes, Perú, pcastillocarrillo@yahoo.es, https://orcid. org/0000-0002-0255-1047.
\end{abstract}

Autor para correspondencia: Pedro Saúl Castillo-Carrillo. Dr. Jefe del Museo de Entomología y Profesor Principal, Facultad de Ciencias Agrarias, Universidad Nacional de Tumbes, Tumbes, Perú, pcastillocarrillo@ yahoo.es, https://orcid.org/0000-0002-02551047

Citación sugerida / Suggested citation: MORÁN-ROSILLO, J. L.; CASTILLOCARRILLO, P. S. 2020. El barrenador del fruto y tallo del cacao (Carmenta theobromae, Lepidoptera: Sesiidae) en el valle de Zarumilla, Tumbes, Perú. Revista Colombiana de Entomología 46 (1): e10165. https://doi. org/10.25100/socolen.v46i1.10165

Recibido: 9-mar-2018

Aceptado: 2-dic-2019

Publicado: 5-ago-2020

Revista Colombiana de Entomología

ISSN (Impreso): 0120-0488

ISSN (En línea): 2665-4385

https://revistacolombianaentomologia.univalle.edu.co/

Open access

Publicadores / Publishers:

Sociedad Colombiana de Entomología

SOCOLEN (Bogotá, D. C., Colombia)

http://www.socolen.org.co

Universidad del Valle (Cali, Colombia)

http://www.univalle.edu.co/

(C) 2020 Sociedad Colombiana de Entomología - SOCOLEN y Universidad del Valle - Univalle
Resumen: Desde el 2009 se ha evidenciado en las plantaciones de cacao del valle de Zarumilla (Tumbes, Perú), la presencia de un insecto plaga de especie aún no identificada, cuyas larvas perforan los tallos y frutos del cacao, y afectan su valor comercial por la invasión de microorganismos patógenos secundarios. Este trabajo de investigación que se realizó de marzo a agosto del 2014 tuvo como objetivos identificar y describir morfológicamente al barrenador del fruto y tallo del cacao, no reportado para las condiciones de este valle. Las recolectas se realizaron en campos cacaoteros del valle de Zarumilla y la crianza de los estados inmaduros, en el laboratorio de Entomología de la Universidad Nacional de Tumbes. Los resultados permitieron determinar que la especie cuya larva perfora los tallos y frutos del cacao es Carmenta theobromae. Se describen las principales características morfológicas de esta especie, referente a los estados de huevo, larva, pupa y adulto de ambos sexos. Además, del material biológico recolectado en campo durante el muestreo, se identificaron dos parasitoides a nivel de familia, un díptero Tachinidae recuperado de larvas y un himenóptero Chalcididae a partir de pupas. Se concluye que la especie que barrena los frutos de cacao en las plantaciones del valle de Zarumilla es $C$. theobromae. El presente estudio servirá de base para realizar futuras investigaciones de la biología y comportamiento en relación con los factores ambientales y determinar si esta especie tiene preferencia por determinados clones de cacao sembrados en la zona.

Palabras clave: Carmenta theobromae, Sesiidae, Lepidoptera, Perú, cacao, plaga.

Abstract: Since 2009, the presence of a pest insect of an unidentified species whose larvae pierce the stems and fruits of the cocoa plant, affecting the commercial value of the fruits by the invasion of secondary pathogenic microorganisms, has been demonstrated in the cocoa plantations of the Zarumilla valley, (Tumbes, Peru). This research work was carried out from March to August 2014 in order to identify and morphologically characterize the borer of the fruit and stem of the cocoa plant not reported for the conditions of this valley. The collections were made in cocoa fields of the Zarumilla valley and the breeding of immature states was carried out in the Entomology laboratory of the National University of Tumbes. The results made it possible to determine that the species whose larva pierces the cacao stems and fruits is Carmenta theobromae. The main morphological characteristics of this species are described, referring to the egg, larva, pupa and adult stages of both sexes. In addition, from the biological material collected in the field during the sampling, it was possible to identify two parasitoids at the family level, a dipteran Tachinidae recovered from larvae and a hymenopteran Chalcididae recovered from pupae. It is concluded that the species that burrows cocoa fruits in cocoa plantations in the Zarumilla Valley is $C$. theobromae. The present study will serve as the basis for biology and behavioral studies of in relation to environmental factors and to determine if this species has a preference for certain cocoa clones planted in the valley.

Keywords: Carmenta theobromae, Sesiidae, Lepidoptera, Peru, cocoa, pest.

\section{Introducción}

En la región Tumbes (Perú), el cultivo del cacao (Theobroma cacao L., 1753), después del banano, es el segundo cultivo bandera exportable de la región, con cultivos de 763 ha a nivel regional y 427 en la provincia de Zarumilla, lo que representa el $56 \%$ de la superficie de cacao instalada en la región. El cacao se cultiva bajo condiciones de temperatura promedio mínima que fluctúa entre 18 y 23 ${ }^{\circ} \mathrm{C}$, y la máxima entre 26 y $36{ }^{\circ} \mathrm{C}$, y humedad relativa oscilando entre 72 a $82 \%$, 
condiciones ambientales favorables para el desarrollo óptimo del cultivo pero también para la presencia de insectos plagas y enfermedades (Gobierno Regional de Tumbes 2013).

En esta provincia, se comenzó a evidenciar desde el 2009 la presencia de un insecto plaga desconocido para la mayoría de los productores cacaoteros de esta región, cuya incidencia se había hecho evidente [comunicación personal de los productores cacaoteros del valle de Zarumilla y personal técnico de la Asociación Regional de Productores de Cacao de Tumbes (ARPROCAT)]. Las larvas de este fitófago perforan los tallos, cojines florales y frutos del cacao (Vásquez et al. 2015), ocasionan galerías al interior de ellos, generan la pérdida del valor comercial del fruto por invasión de microorganismos patógenos secundarios como Phytophthora sp., causante de la pudrición del fruto y, en consecuencia, afectan las semillas (Morillo et al. 2009). A nivel de tallos se produce una reducción de la función de conducción y soporte; pérdida de ramas productoras e injertos, hasta llegar a reducir la producción significativamente (Brown y Mizell 1993; McKern y Szalanski 2007; Mckern et al. 2008).

Delgado (2005) realizó la descripción de adultos e inmaduros de los perforadores del fruto de cacao Carmenta theobromae (Busck, 1910) y Carmenta foraseminis (Eichlin, 1995) (Lepidoptera: Sesiidae), en ejemplares encontrados en la región costera del estado Aragua, Venezuela. El autor inició una cría, en condiciones de laboratorio, con la cual describió las fases de huevo, larva, pupa y adulto de cada especie y, así mismo, propuso la utilización de los caracteres de pupas, a partir de las exuvias encontradas en los frutos perforados en el campo, para diagnosticar la presencia de ambas especies. Así mismo, también observó el comportamiento de la hembra de $C$. theobromae la cual oviposita en la superficie de los frutos tiernos, de donde emergen las larvas que perforan la corteza para alimentarse, forman galerías, ocasionando que se rellenen de excrementos oscuros y apariencia a borra de café, pero sin afectar la parte interna ni los granos, pudiendo incluso aprovecharse. Recientemente, Sarmiento Naizaque et al. (2016) registraron para Colombia a C. theobromae como una importante plaga del cultivo de guayaba, se le detectó en el 2006 y se le denominó "gusano anillador" por formar anillos alrededor del fuste y alimentarse por debajo de la corteza del árbol hasta llegar al cambium vascular. Por otro lado, Carabalí et al. (2015) reportan que C. theobromae pasó de consumir la mazorca y semillas del cacao al guayabo, argumento que concuerda con lo registrado por Harms y Aiello (1995), que consideran que las especies cuyas larvas son perforadoras del fruto tienen un comportamiento aberrante o totalmente diferente al encontrado en la mayoría de las especies de la familia Sesiidae. Las hembras ovipositan sobre grietas en los troncos, ramas o raíces expuestas y cuando eclosionan, sus larvas forman galerías en los lugares donde fueron ovipositados (Hernández-Baz y Equihua 2000). En guayaba, Sarmiento Naizaque et al. (2016) observaron que las ovoposiciones se realizaban principalmente en la corteza del tallo donde las posturas fueron simples, espaciadas y de forma oblonga o elipsoidal.

Es interesante resaltar que los adultos de Sesiidae a la que pertenece $C$. theobromae, incluyen 1.400 especies en 150 géneros de distribución mundial (Heppner y Duckworth 1981; Wang y Li 2008), son polillas de tamaño pequeño que en la cabeza a lo largo del margen posterior tienen una línea de escamas (Scoble 1992), presentan alas hialinas u opacas, elongadas, características que son propias del género Carmenta
Edwards, además de que su abdomen es sésil, bandeado y afinado hacia la cauda, con pelos pequeños; las antenas son engrosadas hacia su extremo (Edwards 1881). Además, estas polillas se caracterizan por tener hábito diurno y patrones de coloración y comportamiento altamente modificados, que resultan semejantes a algunos himenópteros (Duckworth y Eichlin 1977; Wang y Li 2008). Cabe mencionar que Eichlin (1995) reporta que C. theobromae también se ha encontrado atacando semillas de Gustavia superba (H.B.K.) Berg.

De lo descrito se puede destacar que C. theobromae, "barrenador de fruto y tallo del cacao", es trascendente en cultivos de importancia económica. En el presente trabajo se planteó como objetivo identificar la especie y ampliar el conocimiento sobre su morfología de los estados de desarrollo, distribución, y comportamiento en el valle de Zarumilla, Tumbes, Perú, información que permitirá adoptar medidas oportunas de control.

\section{Materiales y métodos}

La investigación se realizó entre marzo y agosto de 2014 recolectando las muestras en parcelas cacaoteras ubicadas en los centros poblados de: Cuchareta Baja, Uña de Gato, El Porvenir, La Palma, El Huaco, La Coja, Los Limos, Pueblo Nuevo, Quebrada Seca y El Tutumo, pertenecientes a la provincia de Zarumilla (Tabla 1). Las muestras fueron llevadas al laboratorio de Entomología de la Universidad Nacional de Tumbes en el campus universitario La Cruz, a 4 $m$ de altitud.

Cada siete días se realizaron visitas de campo a los lugares seleccionados, y en cada punto se tomaron 10 plantas de cacao afectadas por el barrenador del fruto y tallo del cacao de las cuales, con la ayuda de una cuchilla se extrajeron de su interior (tallo y fruto) dos larvas por planta. Estas fueron colocadas de forma individual en el interior de un trozo de tallo o fruto de cacao ubicado en un recipiente plástico para su traslado al laboratorio. En cada visita se recolectaron frutos tiernos y vástagos de cacao para ser usados como alimento. En laboratorio, las larvas recolectadas fueron individualizadas en vasos plásticos de medio kilo, colocando en ellos trozos de tallo y frutos de cacao como alimento el cual era renovado periódicamente cada vez que registraba pérdida de humedad. A partir de este momento se realizaron observaciones diarias para describir y registrar los diferentes

Tabla 1. Ubicación de parcelas cacaoteras muestreadas en la Provincia de Zarumilla, Tumbes, Perú, 2014.

\begin{tabular}{llcc}
\hline \multirow{2}{*}{ Localidad } & \multicolumn{3}{c}{ Ubicación geográfica } \\
\cline { 2 - 4 } & Latitud sur & Longitud oeste & $\begin{array}{c}\text { Altitud } \\
\text { (msnm) }\end{array}$ \\
\hline Cuchareta Baja & $03^{\circ} 34^{\prime} 30^{\prime \prime}$ & $80^{\circ} 15^{\prime} 56^{\prime \prime}$ & 16 \\
Uña de Gato & $03^{\circ} 47^{\prime} 00^{\prime \prime}$ & $80^{\circ} 23^{\prime} 14^{\prime \prime}$ & 20 \\
El Porvenir & $03^{\circ} 45^{\prime} 59^{\prime \prime}$ & $80^{\circ} 17^{\prime} 57^{\prime \prime}$ & 29 \\
La Palma & $03^{\circ} 40^{\prime} 16^{\prime \prime}$ & $80^{\circ} 27^{\prime} 16^{\prime \prime}$ & 23 \\
El Huaco & $03^{\circ} 39^{\prime} 32^{\prime \prime}$ & $80^{\circ} 15^{\prime} 57^{\prime \prime}$ & 36 \\
La Coja & $03^{\circ} 47^{\prime} 13^{\prime \prime}$ & $80^{\circ} 19^{\prime} 28^{\prime \prime}$ & 44 \\
Los Limos & $03^{\circ} 36^{\prime} 29^{\prime \prime}$ & $80^{\circ} 25^{\prime} 53^{\prime \prime}$ & 52 \\
Pueblo Nuevo & $03^{\circ} 38^{\prime} 23^{\prime \prime}$ & $80^{\circ} 18^{\prime} 02^{\prime \prime}$ & 61 \\
Quebrada Seca & $03^{\circ} 41^{\prime} 57^{\prime \prime}$ & $80^{\circ} 23^{\prime} 30^{\prime \prime}$ & 60 \\
El Tutumo & $0^{\circ} 55^{\prime} 46^{\prime \prime}$ & $80^{\circ} 22^{\prime} 22^{\prime \prime}$ & 92 \\
\hline
\end{tabular}


cambios morfológicos sucedidos a través de los estados de desarrollo del insecto. A partir de la observación del orden y disposición de los ganchos de las propatas de las larvas criadas en laboratorio y con la ayuda de claves taxonómicas se realizó la identificación a nivel de orden y familia mientras que para género y especie se utilizaron los caracteres descritos por Delgado (2005). Los adultos machos y hembras recién emergidos fueron congelados por 20 minutos para provocar su muerte, luego se colocaron en una solución de hidróxido de potasio al $10 \%$ por espacio de 24 horas. Con ayuda de pinzas y bisturí se hizo la disección del abdomen, luego se extrajo la genitalia masculina, se abrió y fotografió desde varios ángulos. A partir de esto se realizó la identificación por comparación. Finalmente, se les puso en micro viales con alcohol para su conservación. Las fotografías fueron enviadas al especialista Dr. Franz Pühringer en Austria. También fueron enviados dos ejemplares hembras y dos machos para corroborar la identificación a través de la secuenciación de ADN. El status taxonómico se completó con la ayuda de las claves desarrolladas por Heppner y Duckworth (1981) y Duckworth y Eichlin (1977). La descripción morfológica y la toma de las dimensiones de huevos, y las diferentes estructuras y apéndices de los estados de huevo, larva, pupa y, adulto, se realizó con ayuda de un estereoscopio Olympus modelo SZ21 con ocular micrométrico, con una platina micrométrica. Para la descripción del huevo se utilizó una muestra de 20 huevos ovipositados por adultos criados en laboratorio a partir de larvas recolectadas en campo. Se analizaron las variables: forma, tamaño y color del corion. En el caso de la forma del huevo, se realizaron comparaciones con reportes en la literatura y para el tamaño se midió el ancho y longitud con el estereoscopio con ocular micrométrico. También se identificaron plantas de cacao con síntomas de perforación en tallos o frutos y se recolectaron trozos de la corteza del tallo o fruto cercana a la zona afectada, los que fueron llevados al laboratorio donde con estereoscopio, se realizó la búsqueda de huevos. Se utilizaron diez larvas del cuarto estadio criadas en laboratorio para las descripciones y se tuvo en cuenta: forma, color, ancho de la cápsula cefálica, tórax y abdomen, disposición de las propatas ventrales y caudales, se consideró observar solo este estadio porque en él se encuentran presentes las estructuras de importancia taxonómica necesarias para la confirmación de la especie. En la descripción de la pupa, se utilizaron aquellas obtenidas en laboratorio en un número de diez, a las que se les observó: forma, tamaño, color y el sexo; se tomó como carácter de referencia la fusión de los segmentos terminales del abdomen (Leskey y Bergh 2003). La descripción de los adultos se realizó con diez ejemplares hembras y diez machos obtenidos a partir de la cría en laboratorio, fueron montados en alfileres entomológicos para describir sus características morfológicas y sus genitalias. Cabe indicar que en este caso no se tomaron datos numéricos para los estados de desarrollo, porque no se había considerado en este estudio realizar estudios de biología.

Análisis estadístico. Se utilizaron los promedios obtenidos en base al número de ejemplares para la determinación de la desviación y error estándar. No se utilizó ningún diseño experimental, porque el objetivo del trabajo fue la identificación taxonómica de la especie del género Carmenta.

\section{Resultados}

Identificación de la especie. Se determinó que Carmenta theobromae (Busck, 1910) (Lepidoptera: Sesiidae) es la especie que barrena el fruto y tallo de cacao en las plantaciones de cacao en el valle de Zarumilla, Tumbes, Perú. Ésta fue descrita inicialmente como Sesia theobromae a partir de un macho emergido de un fruto de cacao colectado en Trinidad y Tobago y que Eichlin (1995) lo ubicó como C. theobromae n. comb.

Descripción morfológica de los estados de desarrollo. Los huevos, miden en promedio 0,51 $\pm 0,007 \mathrm{~mm}$ de longitud por $0,35 \pm 0,013 \mathrm{~mm}$ de ancho (Tabla 2), color marrón claro, forma semirectangular, más largos que anchos con la región anterior redondeada y la posterior roma (Fig. 1A). La superficie dorsal del corion es reticulada, surcada por estructuras blanquecinas en alto relieve a manera de tricomas (Fig. 1B). Ventralmente, es ligeramente cóncava y lisa (Fig. 1C). Los huevos son ovipositados individualmente sobre frutos y tallos de cacao.

Las larvas son eruciformes, color cremoso, cuerpo cilíndrico cubierto de setas marrones, con cinco pares de propatas abdominales, cuatro ubicadas en los segmentos A3 al A6 y un par en el último segmento (Fig. 2A). La cabeza es marrón, ovalada en vista dorsal, tan ancha como el pronoto debido al ensanchamiento de las genas (Fig. 2B), los ocelli,

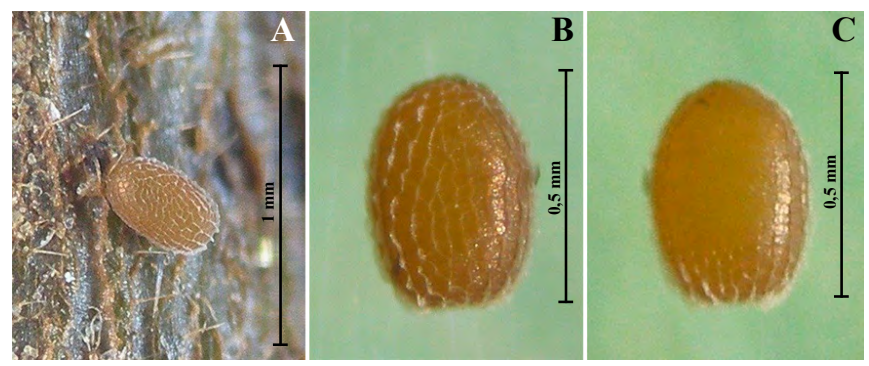

Figura 1. Huevo de Carmenta theobromae: A. En tronco de árbol. B. Vista dorsal. C. Vista ventral.

Tabla 2. Descripción morfométrica de los estados de desarrollo de Carmenta theobromae. Provincia de Zarumilla, Tumbes, Perú, 2014.

\begin{tabular}{|c|c|c|c|c|c|c|}
\hline \multirow{4}{*}{ Dimensiones (mm) } & \multicolumn{6}{|c|}{ Estado de desarrollo } \\
\hline & \multirow{3}{*}{ Huevo $(\mathbf{n}=\mathbf{2 0})$} & \multirow{3}{*}{$\begin{array}{l}\text { Larva (IV estadio) } \\
\qquad(\mathbf{n}=\mathbf{1 0})\end{array}$} & \multirow{2}{*}{\multicolumn{2}{|c|}{$\begin{array}{c}\text { Pupa }(n=10) \\
\text { Sexo }\end{array}$}} & \multirow{2}{*}{\multicolumn{2}{|c|}{$\begin{array}{c}\text { Adulto }(n=10) \\
\text { Sexo }\end{array}$}} \\
\hline & & & & & & \\
\hline & & & Macho & Hembra & Macho & Hembra \\
\hline Ancho & $0,35 \pm 0,013$ & & $2,34 \pm 0,15$ & $2,45 \pm 0,15$ & & \\
\hline Largo & $0,51 \pm 0,007$ & $14,33 \pm 0,13$ & $9,39 \pm 0,55$ & $9,97 \pm 0,37$ & $9,40 \pm 0,41$ & $8,30 \pm 0,42$ \\
\hline Ancho cápsula cefálica & & $1,40 \pm 0,019$ & & & & \\
\hline Longitud expansión alar & & & & & $16,50 \pm 0,46$ & $16,70 \pm 0,84$ \\
\hline
\end{tabular}


ojos simples o stemmata, se encuentran ubicados seis a cada lado de la base de las mandíbulas. El clípeo de forma semirectangular, un poco más ancho que el labro, sin setas. El labro es semirectangular (Fig. 2C). Las mandíbulas (Fig. 2D), se ubican debajo del labro, color marrón oscuro, presentan cinco dientes agudos en su parte distal. Las maxilas (Fig. 2E), de aspecto robusto, fuertes y membranosas. El labium, en la parte inferior, cierra la cavidad preoral, algo transparente de forma rectangular y está ubicado entre las maxilas (Fig. 2E). El tórax es de color cremoso, en el aspecto ventral de cada segmento presenta un par de patas cortas (Fig. 2F). El abdomen de color cremoso de forma, cónica, presenta diez segmentos con los tergos de forma rectangular cubiertos con setas marrones (Fig. 2G), ventralmente lleva cinco pares de propatas (Fig. 2H), cuatro en los segmentos A3-A6 y un par en el segmento A10, las propatas presentan un par de bandas transversales de ganchos uniordinales (Fig. 2I). La longitud de la larva del IV estadio y el ancho de la cápsula cefálica se presentan en la Tabla 2.
La pupa es elíptica, obtecta, marrón oscuro (Fig. 3A), en los machos mide en promedio $9,39 \pm 0,55 \mathrm{~mm}$ de largo y $2,34 \pm 0,15 \mathrm{~mm}$ de ancho, en las hembras $9,97 \pm 0,37$ y 2,45 $\pm 0,19 \mathrm{~mm}$ (Tabla 2). En el tórax, el protórax, tiene aspecto rectangular y está cubierto densamente de puntuaciones, el mesotórax, segmento más desarrollado abarca casi el $70 \%$ de la superficie dorsal (Fig. 3B). El abdomen, consta de diez segmentos (Fig. 3C), en machos con los segmentos A8-A10 fusionados, presentan tres filas de espinas dorsales (Fig. 3D), en la hembra presenta los segmentos A7-A10 fusionados, y cuatro filas de espinas (3E). El ápice del abdomen con ochos espinas, esclerotizadas que siguen un arreglo circular (Fig. 3F). En pupas machos, la región genital está más cerca de la región anal (Fig. 3G), mientras que en hembras está más distante.

$\mathrm{El}$ adulto es una polilla marrón amarillo, con predominio del amarillo en su cuerpo, su aspecto es parecido al de una avispa (Figs. 4A y 4B). El macho ligeramente más grande que la hembra (Fig. 4A; Tabla 1) se diferencian, porque poseen un penacho de escamas apicales en el abdomen

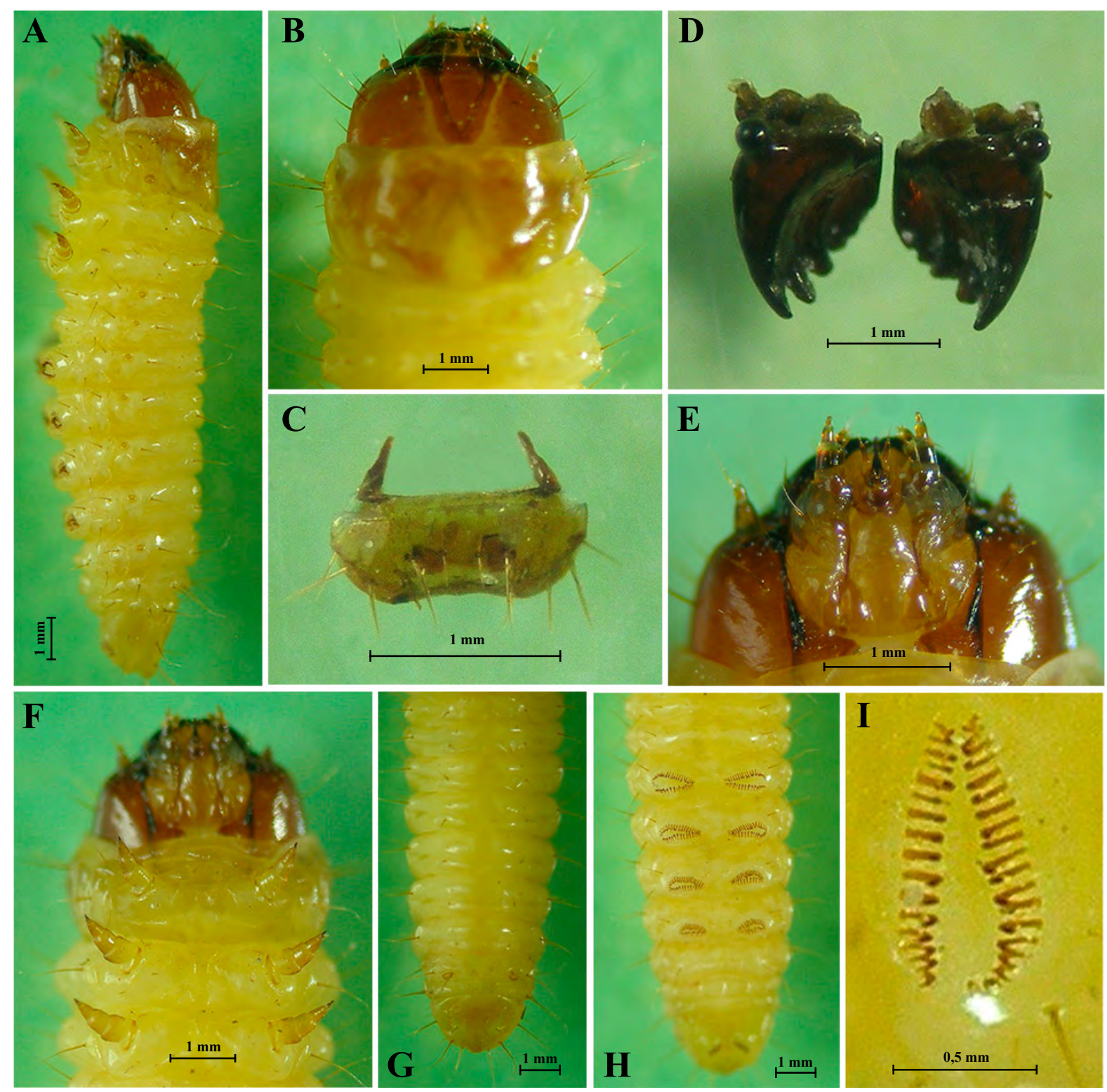

Figura 2. Larva de Carmenta theobromae: A. Vista lateral. B. Cabeza en vista dorsal. C. Labro. D. Mandíbulas. E. Máxilas y labium. F. Patas torácicas. G. Segmentos abdominales. H. Propatas. I. Ganchos. 

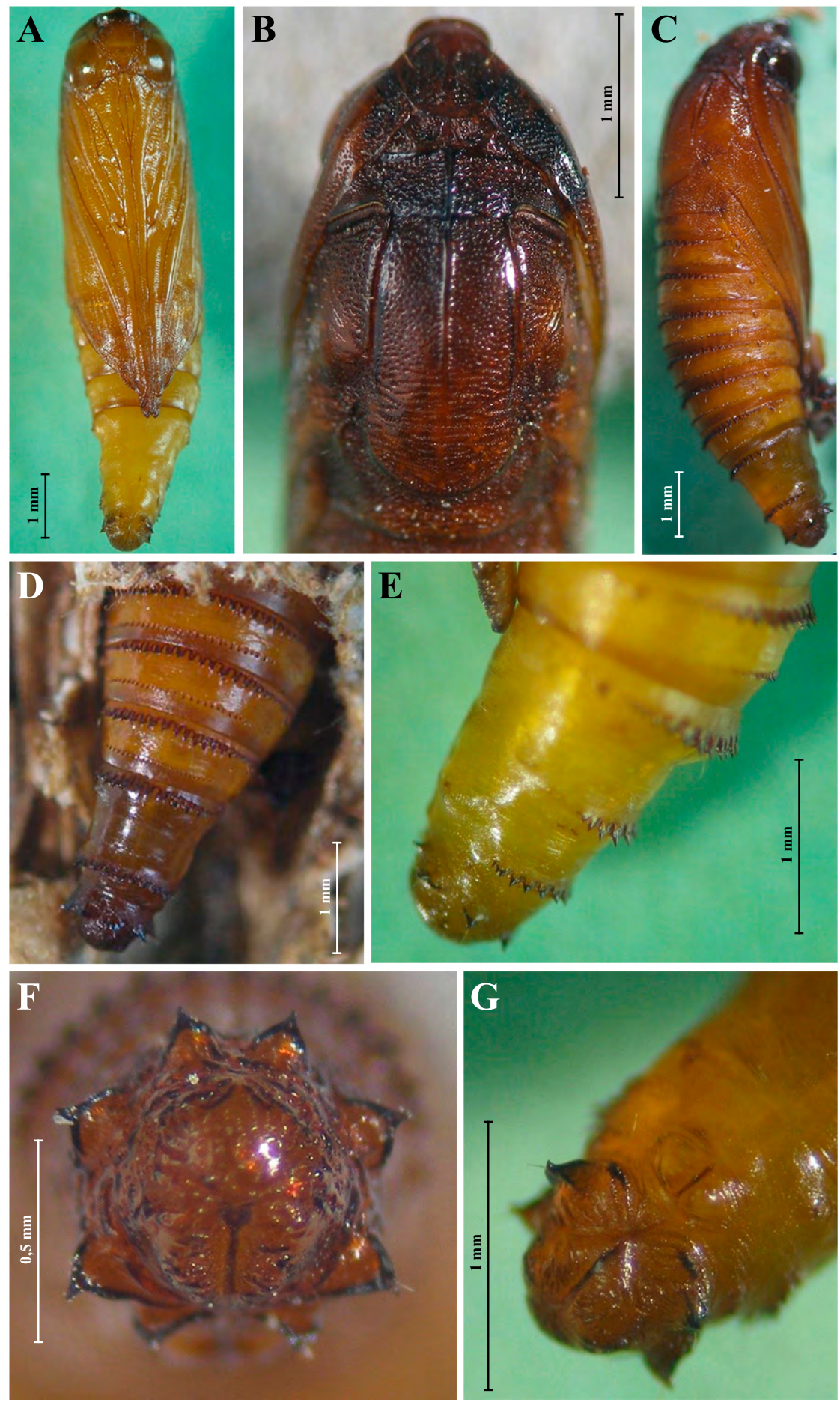

Figura 3. Pupa de Carmenta theobromae: A. Vista ventral. B. Tórax. C. Vista lateral. D. Segmentos terminales del abdomen fusionados en machos con tres hileras de espinas. E. Segmentos terminales abdominales fusionados en hembras con cuatro hileras de espinas. F. Ápice en pupa de macho mostrando espinas anales. G. Región anal y genital en pupa de macho.

y presentan pelos finos y cortos a todo lo largo del borde interno de las antenas, cabeza, es globular, con dos ojos compuestos grandes y un par de ocelos pequeños color negro, ubicados a cada lado del mesón; probóscide larga y labra. Palpos labiales tri-segmentados, curvados hacia arriba, ventralmente cubiertos de escamas amarillas y en su parte apical con algunas de color amarillo. Antenas multisegmentadas, clavadas, dorsalmente oscuras y ventralmente amarillas. El protórax dorsalmente rectangular, angosto, con escamas marrones en su aspecto anterior y escamas amarillas en el posterior delimitando el segmento (Fig. 5A). Las alas anteriores (Fig. 5B), son rectangulares, hialinas, con escamas presentes en los márgenes y las posteriores (Fig. 5C) son translucidas, con escamas amarillas en el margen costal y anal, venas marrones. Frenulum (Fig. 5D), bien desarrollado, en el macho está formado por una sola cerda larga y en la hembra por dos cerdas finas. Patas anteriores (Fig. 5G) con coxas color amarillo, fémur marrón, tibias cortas con 

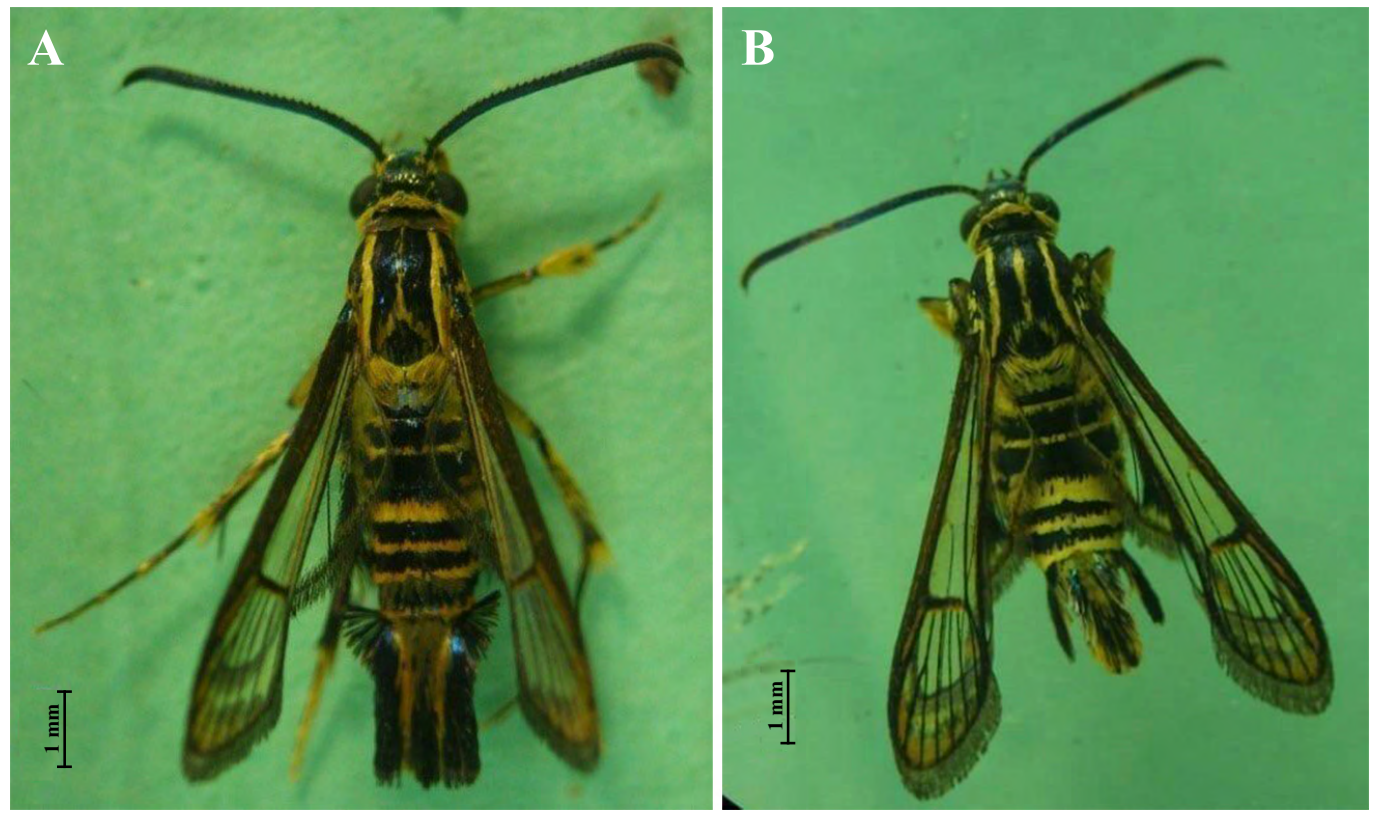

Figura 4. Adulto de Carmenta theobromae: A. Macho. B. Hembra.

escamas amarillas, en su parte ventral distal presenta un penacho de escamas amarillas cubriendo la epífisis, posee cinco tarsómeros y un pretarso con uña bífida. Las patas medias (Fig. 5H), con escamas marrón en las coxas, fémur y tibias marrón ventralmente con escamas amarillas, un pretarso con uña bífida. Patas posteriores marrón (Fig. 5I); el fémur es corto aproximadamente de la mitad del tamaño de la tibia; tibias grandes marrones en el dorso y ventralmente amarillas, terminan en un penacho de finas escamas marrones, presentan dos pares de espinas, una grande y una pequeña, el primer par ubicado en la parte media y el segundo en la parte distal cubierta con el penacho de escamas, tarso con cinco tarsómeros. El abdomen presenta diez segmentos, superficie de notos son más o menos rectangulares, marrones, con bandas transversales estrechas amarillas en la parte posterior, existe dimorfismo sexual en el número de segmentos; en el macho se distinguen siete segmentos visibles y tres fusionados formando el órgano genital (Fig. 5E) y en la hembra presenta seis segmentos visibles y cuatro fusionados forman el órgano genital (Fig. 5F). La genitalia masculina (Fig. 5J) incluye los segmentos abdominales A8-A10, está formada por varias estructuras; las valvas son órganos pares, y los más prominentes, en conjunto forman un estuche que protege y encierra a los demás órganos genitales, el cual está formado
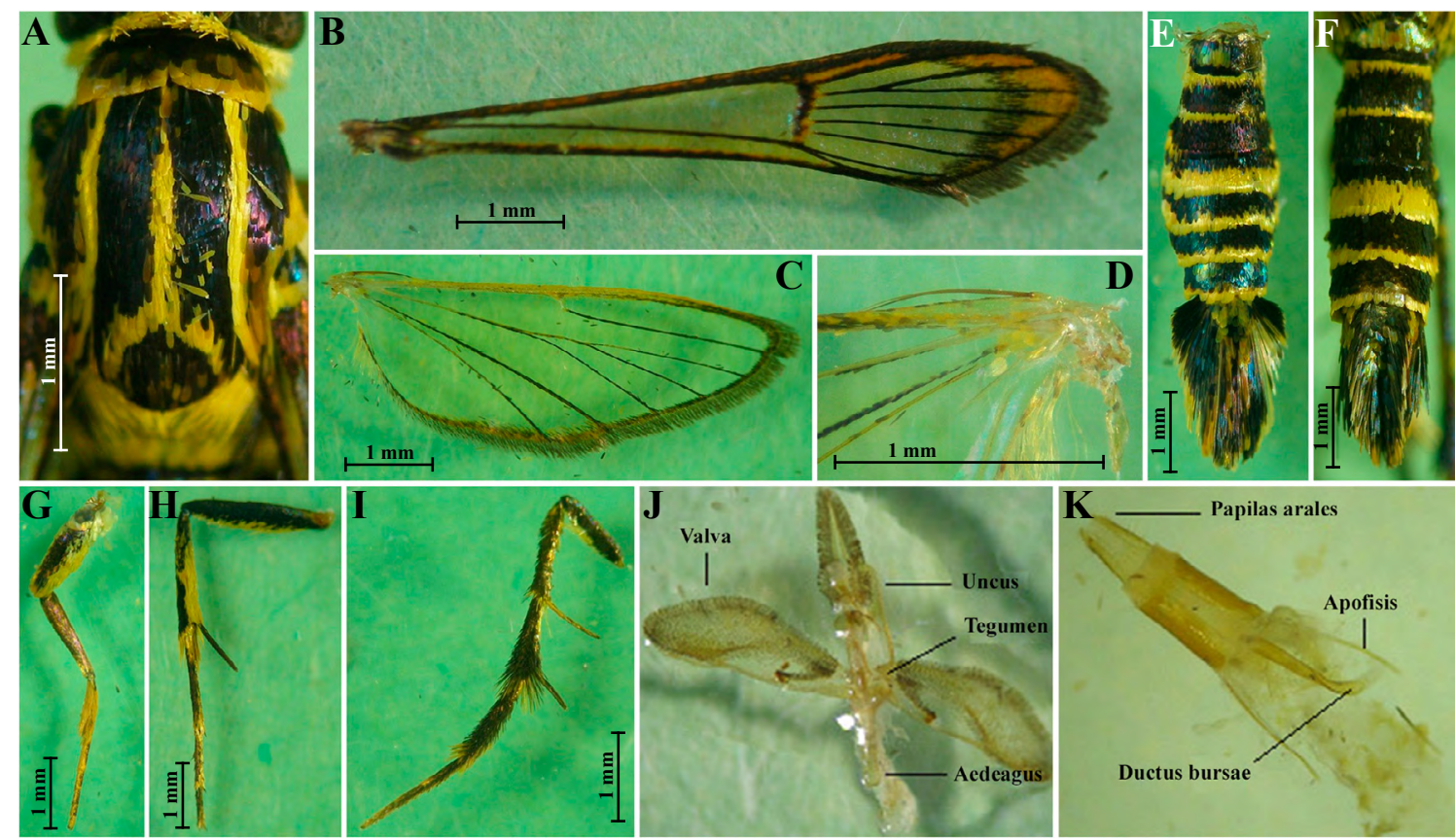

Figura 5. Carmenta theobromae: A. Tórax vista dorsal. B. Ala anterior. C. Ala posterior. D. Frenulum. E. Abdomen de macho. F. Abdomen de hembra. G. Pata anterior. H. Pata media. I. Pata posterior. J. Vista ventral de genitalia masculina. K. Vista ventral de genitalia femenina. 
por pelos laminares dando lugar a un órgano cóncavo. La genitalia femenina (Fig. 5K) es mucho más sencilla que la del macho, incluye los segmentos abdominales A7-A10. Los últimos segmentos son atenuados y forman un tubo delgado telescópico de color claro.

Comportamiento. Los adultos de $C$. theobromae, exhiben hábitos diurnos se les observa durante las horas de la tarde posados a la altura de la base del tallo; presentan mimetismo, sus bandas amarillas estrechas en el noto de los segmentos abdominales dan la apariencia de una avispa del género Polybia (Vespidae). La hembra en el interior de su abdomen, puede contener hasta 80 huevos, oviposita de forma individual sobre los frutos, a la altura de la base del tallo, ramas de cacao y en las heridas causadas por herramientas de labranza sobre los tallos y ramas. Una vez eclosionado el huevo, la larva perfora la corteza de tallos y ramas y se introduce al interior de la corteza produciendo galerías dejando sus excrementos color marrón al exterior, cuando el daño es severo en ramas jóvenes e injertos, éstas se caen o se debilitan produciendo una disminución del área foliar y la muerte del injerto. En frutos, las larvas atraviesan el epicarpio y se alimentan del mesocarpio, formando galerías, no atraviesan el mesocarpio por lo que las semillas están intactas pudiéndose cosechar, el daño en frutos es indirecto, debido a que a través de las heridas causadas por las larvas se produce el ingreso de microorganismos que pueden provocar la pudrición parcial del fruto. En un fruto se pueden encontrar varias larvas, una vez completado su desarrollo empupan en el interior del fruto o dentro del tallo, dejando parte de la exuvia pupal expuesta al exterior cuando emergen.

Otras observaciones. De las larvas y pupas recolectadas en campos de cacao, se recuperaron dos parasitoides, un díptero Tachinidae (Fig. 6A) en larvas de C. theobromae, este hecho se observó en una larva de El Tutumo, distrito de Matapalo, el cocón de esta larva fue revisado, encontrando en su interior un pupario del parasitoide con los residuos de la larva (Fig. 6B) e igualmente de una pupa de $C$. theobromae recolectada en la localidad de Uña de Gato se recuperó un himenóptero Chalcididae (Fig. 6C). En la zona del Huaco se pudo observar in situ, a ejemplares de este calcídido no identificado revoloteando cerca de tallos atacados por C. theobromae.

\section{Discusión}

La identificación de la especie realizada con la ayuda de las claves desarrolladas por Heppner y Duckworth (1981) y Duckworth y Eichlin (1977), fue corroborada por el especialista austriaco, Dr. Franz Pühringer.

En lo que corresponde a las descripciones morfológicas con relación a las dimensiones de los huevos de C. theobromae, los resultados de Delgado (2005) para Venezuela, (2,86 x 1,93 $\mathrm{mm})$, difieren con los obtenidos en este estudio, pero están dentro de los rangos encontrados por Sarmiento Naizaque et al. (2016) quienes registraron en promedio 0,22 $\pm 0,012 \mathrm{~mm}$ de ancho y $0,37 \pm 0,011 \mathrm{~mm}$ de longitud $(\mathrm{n}=15)$ e igualmente coinciden con las descripciones para la forma indicando que es oblonga o elipsoidal y que presenta 20 costas radiales que se observan a manera de estrías y las celdas del corion exhiben un modelo poligonal. En relación al IV estadio larval estudiado la longitud obtenida $(14,33 \pm 0,13 \mathrm{~mm})$ es muy semejante a lo citado por Sarmiento Naizaque et al. (2016) (14,29 $\pm 0,25$ $\mathrm{mm}$ ) y en lo que corresponde al ancho de la cápsula cefálica $(1,40 \pm 0,019 \mathrm{~mm})$ en nuestro estudio, se encuentra dentro de los rangos encontrados por estos autores (1,36 - 1,47 mm). En relación al estado de pupa las descripciones morfológicas son concordantes con las reportadas por Delgado (2005). En cambio con este mismo autor para los adultos se presentan algunas diferencias, ya que describe una banda ancha de color amarillo en el segmento A5, mientras que en nuestro estudio se encontró en el segmento A4.

Los resultados en cuanto a daños causados por $C$. theobromae, en frutos de cacao concuerdan con los encontrados para Venezuela por Vivas et al. (2005) y Morillo et al. (2009) quienes indican que las larvas producen perforaciones y galerías internas en el pericarpio de fruta infestada, y el daño conduce a otro secundario debido a infecciones fúngicas producidas por hongos como Phytophthora sp., que hacen que la fruta sufra un proceso de descomposición, lo característico es que se puede observar pilas de excrementos de color marrón rojizo, con el aspecto de café molido, unidas por una masa de hilos de seda a la entrada de la perforación realizada por la larva. Por otro lado, Vásquez et al. (2015) para Colombia en un cultivo de cacao con el objetivo de registrar la ocurrencia simultánea de $C$. theobromae con $C$. foraseminis, y el daño de $C$. theobromae en un órgano diferente a la mazorca de cacao, encontraron a $C$. theobromae atacando el tallo y cojines florales de cacao de los clones CCN 51, TSH 812, ICS 1 e ICS 39, los cuales presentaban un crecimiento anormal, cuyo síntoma era similar a la enfermedad denominada agalla floral del cacao. En nuestro caso no se encontró daño en cojines florales. Respecto al comportamiento de los adultos se ha observado lo destacado por Herrera et al. (2012) en Venezuela para determinar y comparar el comportamiento de estas especies referente a que estos insectos son de hábitos diurnos y miméticos.
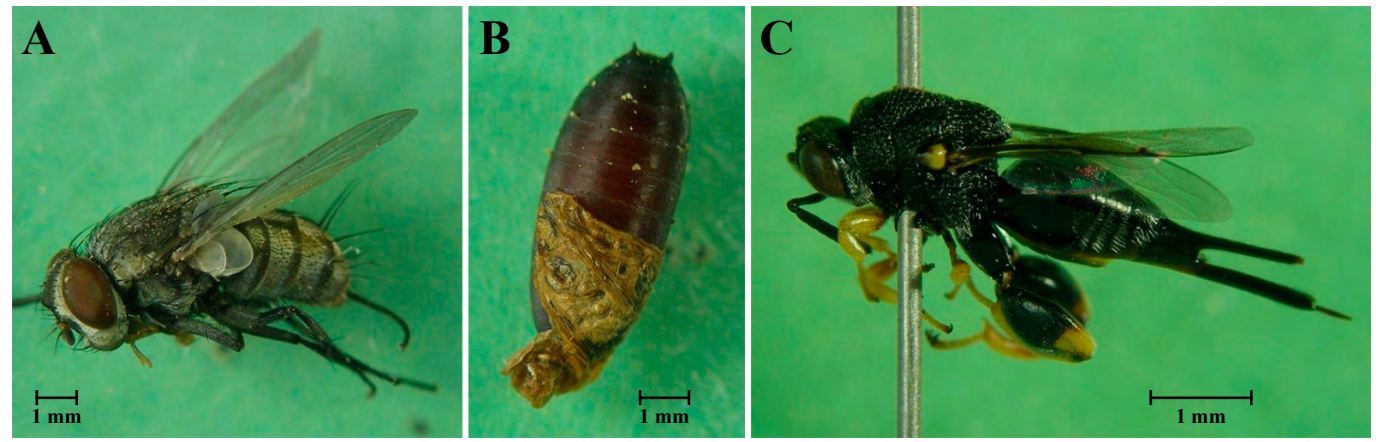

Figura 6. Parasitoides de Carmenta theobromae: A. Adulto de la familia Tachinidae. B. Pupario del taquínido. C. Adulto de la familia Chalcididae. 
En relación a los parasitoides encontrados en el estudio, Carabalí et al. (2015) también registran para Colombia parasitismo de hasta $60 \%$ de pupas por dos especies de Chalcididae del género Brachymeria: B. pedalis y B. conica, y dos especies de Tachinidae no identificadas de los géneros Siphostumia y Sturmiomima.

\section{Conclusiones}

El perforador del fruto y tallo del cacao presente en varias plantaciones de cacao del valle de Zarumilla, Tumbes, Perú, se corresponde con la especie Carmenta theobromae (Lepidoptera: Sesiidae). Este insecto se presenta perforando tallos y frutos de cacao, produciendo galerías en su interior, hecho que genera la pérdida comercial del fruto. Así mismo se evidencia que este insecto plaga tiene enemigos naturales, ya que durante los muestreos realizados se recuperó un taquínido y un calcídido. La realización de este trabajo ha aclarado las dudas que se tenían sobre la especie, y permite indicar que por ahora el valle de Zarumilla, está libre de C. foraseminis (Busck) Eichlin, una especie cuyos daños son más severos e importantes. En relación a la especie identificada se requiere realizar una mayor investigación referente a su biología en relación con los factores ambientales y al material varietal con que se cuenta. Por otro lado, se requiere ampliar la prospección de enemigos naturales y los aspectos relacionados con atrayentes sexuales, bien sea feromonas o kairomonas, de tal manera que permitan generar estrategias de lucha de reducido impacto ambiental contra esta especie, más aún cuando los tipos de cultivos establecidos tienden a incrementar el área de cultivos orgánicos en la región.

\section{Agradecimientos}

Los autores expresan su agradecimiento a la Universidad Nacional de Tumbes, la cual financió la investigación a través del Concurso de Proyectos de Tesis de Pregrado para Alumnos y Bachilleres de la Universidad Nacional de Tumbes 2014, aprobado con resolución No 0564-2014/UNT-R. Al Dr. Franz Pûhringer por la identificación de la especie.

\section{Literatura citada}

BROWN, L. N.; MIZELL, R. F. III. 1993. The clearwing borers of Florida (Lepidoptera: Sesiidae). Tropical Lepidoptera 4 (suppl. 4): $1-21$.

CARABALÍ, A.; INSUASTY, O.; PULIDO, V.; CANACUÁN, D. 2015. Insectos. Plagas de importancia económica en el cultivo de la guayaba y sus estrategias de control. Bogotá, Colombia: Corpoica. 76 p. Disponible en: https://repository.agrosavia. co/bitstream/handle/20.500.12324/13139/76989_66413. pdf?sequence $=1 \&$ isAllowed $=y$ [Fecha revisión: 30 enero 2018].

DELGADO, N. 2005. Caracterización morfológica de los Sesiidae (Insecta: Lepidoptera) perforadores del fruto del cacao (Theobroma cacao L.), presentes en la región costera del estado Aragua, Venezuela. Entomotropica 20 (2): 97-111.

DUCKWORTH, W.; ECHLIN, T. 1977. A classification of the Sesiidae of America North of Mexico (Lepidoptera: Sesioidea). Occasional papers in Entomology, $\mathrm{N}^{\circ}$ 026, State of California, Department of food and agriculture, division of plant industrylaboratory services. $55 \mathrm{p}$.

EDWARDS, H. 1881. New genera and species of the family Aegeridae. Papilio 1 (10): 179-208. https://doi.org/10.5962/bhl. part. 23310

EICHLIN, T. 1995. A new clearwing moth (Sesiidae: Sesiinae). Journal of the Lepidopterists'Society 49 (1): 39-42.
GOBIERNO REGIONAL DE TUMBES. 2013. Guía Metodológica del cultivo de cacao en la Región Tumbes. Primera edición. 89 p.

HARMS, K.; AIELLO, A. 1995. Seed boring by tropical clearwing moths (Sesiidae): Aberrant behavior or widespread habit. The Journal of the Lepidopterist' Society 49 (1): 43-48.

HEPPNER, J. B.; DUCKWORTH, W. D. 1981. Classification of the superfamily Sesioidea (Lepidoptera: Ditrysia). Smithsonian Contributions to Zoology No 314.144 p. https://doi.org/10.5479/ si.00810282.314

HERNÁNDEZ-BAZ， F.; EQUIHUA， A. 2000. Apuntes de Entomología Forestal II. (Dampf) (Lepidoptera: Sesiidae) Una nueva plaga forestal para el estado de Veracruz, México. Foresta Veracruzana 2 (2): 59-62.

HERRERA, B.; MORILLO, F.; SAINZ, C.; LIENDO, C.; FLORES, G.; HERNÁNDEZ, J. V. 2012. Comportamiento de los perforadores del fruto de cacao, Carmenta theobromae (Busk) y Carmenta foraseminis Eichlin (Lepidoptera: Sesiidae). Entomología Mexicana 11 (1): 386-390.

LESKEY, T. C.; BERGH, J. C. 2003. A simple character for sex differentiation of pupae and pupal exuviae of the dogwood borer (Lepidoptera: Sesiidae). Florida Entomologist 86 (3): 378-380. https://doi.org/10.1653/0015-4040(2003)086[0378:ASCFSD]2. $0 . \mathrm{CO} ; 2$

McKERN, J. A.; SZALANSKI, A. L. 2007. Molecular diagnostics of economically important clearwing moths (Lepidoptera: Sesiidae). Florida Entomologist 90 (3): 475-479. https://doi. org/10.1653/0015-4040(2007)90[475:MDOEIC]2.0.CO;2

McKERN, J. A.; SZALANSKI, A. L.; JOHNSON, D. T.; DOWLING, A. P. G. 2008. Molecular phylogeny of Sesiidae (Lepidoptera) inferred from mitochondrial DNA sequences. Journal of Agricultural and Urban Entomology 25 (3): 165-177. https://doi.org/10.3954/1523-5475-25.3.165

MORILLO, F.; SÁNCHEZ, P.; HERRERA, B.; LIENDOBARANDIARAN, C.; MUÑOZ, W.; HERNÁNDEZ, J. V. 2009. Pupal development, longevity and behavior of Carmenta theobromae (Lepidoptera: Sesiidae). Florida Entomologist 92 (2): 355-361. https://doi.org/10.1653/024.092.0222

SARMIENTO NAIZAQUE, Z. X.; INSUASTY BURBANO, O. I.; MARTÍNEZ OSORIO, J. W.; BARRETO-TRIANA, N. 2016. Aspectos biológicos del anillador de la guayaba Carmenta theobromae (Lepidoptera: Sesiidae) en Santander, Colombia. Revista Colombiana de Entomología 42 (2): 176-183. https:// doi.org/10.25100/socolen.v42i2.6689

SCOBLE, M. J. 1992. The Lepidoptera form, function and diversity. The Natural History Museum in association with Oxford University Press $404 \mathrm{p}$.

VÁSQUEZ, Y.; MUÑOZ, J.; MURIEL, S.; HERNÁNDEZ, F. 2015. Ocurrencia de los barrenadores (Carmenta foraseminis Eichlin y Carmenta theobromae (Busck) (Lepidoptera: Sessidae) en Theobroma cacao L., en el Departamento de AntioquiaColombia. Boletín del Museo de Entomología de la Universidad del Valle 16 (1): 34-38.

VIVAS, A.; SÁNCHEZ, L.; MONCADA, A.; MÁRQUEZ, D. 2005. Cacao: aspectos agronómicos para su cultivo. Fundación para el Desarrollo de la Ciencia y la Tecnología del Estado de Táchira, Táchira, Venezuela. $40 \mathrm{p}$.

WANG, Q.; LI, H. 2008. Catalogue of the family Sesiidae in China (Lepidoptera: Sesiidae). SHILAP Revista de Lepidopterología 36 (144): 507-526.

\section{Origen y financiación}

El presente trabajo de investigación fue financiado por la Universidad Nacional de Tumbes con recursos ordinarios de las fuentes de Canon y Sobre Canon.

\section{Contribución de los autores}

José Luis Morán Rosillo: Toma de muestras en campo, crianza $y$ registro de datos en laboratorio, análisis y escritura de resultados y discusión.

Pedro Saúl Castillo Carrillo: Supervisión del trabajo en campo y laboratorio, revisión y edición del documento final. 